\title{
Efficacy and functional outcome of bone marrow aspirate concentrate injection in osteoarthritis of knee-A prospective study
}

\author{
J. Tharik Ajees ${ }^{1, *}$, Mohan Kumar $\mathbf{M}^{2}$ \\ ${ }^{1}$ Post Graduate, ${ }^{2}$ Professor, Dept. of Orthopaedics, Sri Ramachandra Medical College and Research Institute, Chennai, Tamil \\ Nadu, India
}

*Corresponding Author: J. Tharik Ajees

Email: kirubasam01@gmail.com

\begin{abstract}
Introduction: Primary osteoarthritis is a very common progressive disease which usually ends up in deformities and morbidity. Osteoarthritis usually presents with joint pain, tenderness, limitation of movement, crepitus. BMAC can be useful in reducing pain and improve functional outcome with also in progressive of disease.

Materials and Methods: This is an observational study conducted during the period of April 2015 to June 2017 on 30 samples. The descriptive statistical analysis was performed. The surgical procedure of Bone marrow aspirate concentration was carried out. Evaluation of Pre-Operative and Post-Operative functional outcome.

Results: In this 19 (63.3\%) patient presented with left knee osteoarthritis and 11 (36\%) patients right knee osteoarthritis. 19 patients presented with grade 2 osteoarthritis and 11 patients presented with grade 3 osteoarthritis. VAS pain score $86.6 \%$ were in score range of 3-4 preoperatively which decreased to score range of 0-2 100\% of the patients after BMAC Injection postoperatively at 6 months follow up.

Conclusion: The use of Bone marrow concentrate injection (BMAC) in Osteoarthritis of knee was found to be more efficacious with good clinical and functional outcome.
\end{abstract}

Keywords: Osteoarthritis, BMAC, WOMAC score, Knee society score.

\section{Introduction}

Primary Osteoarthritis (OA) is a very common chronic disease in India. Knee is the common site for osteoarthritis associated with usually various deformity. Osteoarthritis is a result of both mechanical and biologic events that destabilize synthesis of articular cartilage chondrocytes and extracellular matrix, and subchondral bone. $^{1}$

Osteoarthritis usually presents with joint pain, tenderness, limitation of movement, crepitus. Osteoarthritis is usually classified as primary (idiopathic) or secondary may be due to trauma, underlying joint disorders, metabolic disorders and neuropathic joints. ${ }^{2}$ Tibiofemoral Osteoarthritis is found to be more common than patellofemoral Osteoarthritis. Increased body weight, age, genetic factors, meniscectomy are few common risk factor for developing early Osteoarthritis knee. ${ }^{3}$

In general, joint space loss becomes apparent radiographic finding. After loss of joint space, sclerosis becomes more prominent, and subchondral cyst formation is an important finding in Osteoarthritis. Further radiologically Osteoarthritis knee can be classified into 4 grades with Kellegren-Lawrence scoring system. ${ }^{4}$ Lateral wedge foot orthoses and addition of a subtalar strap to a lateral-wedge insole are useful for reducing the load on the medial compartment. But, still progression of disease cannot be controlled. High tibial osteotomy and unicondylar replacement are known to have good symptomatic and functional outcome but still most of the patients end up in Total knee replacement.
Bone marrow aspiration concentrate (BMAC) may possess a high potency for chondrogenic, osseous healing because it also stem cells and multiple growth factors. BMAC is obtained through density gradient centrifugation of bone marrow typically aspirated from the iliac crest. Similar to PRP, BMAC contains platelets, but also enriched with growth factors, progenitor cells and mesenchymal stem cells. Which is known to have healing properties as well as anti-inflammatory properties. Since osteoarthritis is a very common progressive disease which usually ends up in deformities and morbidity. BMAC can be useful in reducing pain and improve functional outcome with also in progressive of disease. $^{5}$

\section{Objectives}

To study efficacy and functional outcome of bone marrow concentrate injection in osteoarthritis of knee.

\section{Materials and Methods}

The current study is an observational prospective study. The study duration was April 2015 to June 2017. This study was undertaken in Orthopaedic department, Sri Ramachandra Medical College and Research Institute. The study population was 30 . To describe about the data descriptive statistics, frequency analysis, percentage analysis Epi info software package was used. Inclusion Criteria: All primary osteoarthritic patients > 50 years of age diagnosed as osteoarthritis as per American rheumatoid criteria and Osteoarthritis of knee type II and III as per Kellgren Lawrence classification. 
Exclusion Criteria: Patient received HC injection or viscosupplements, Patient with ongoing infectious disease, Patients with secondary OA, Osteoarthritis of knee type IV and All inflammatory arthritis and neuropathic arthritis.

\section{Surgical Technique \\ Bone marrow Concentrate Preparation: The Bone} Marrow Aspirate Concentrate protocol involves two separate but related procedures. Firstly the aspiration procedure Performed by a physician and assisted by a scrub nurse under short General anaesthesia. Followed by the concentration procedure. Performed by a circulating nurse. Bone marrow concentrate is prepared using Harvest system (centrifuge machine)

Patient Preparation: Positioning the patient in the operation theatre based on the approach to be used for the Bone Marrow Aspiration - posterior. Bone Marrow Aspiration procedures are typically performed under short general anaesthesia. Along with that locally we Injected 2-4 mL of $1 \%$ Lidocaine intradermally on each side of the iliac crest where the puncture is planned. Scrub the portion from the lower back to the upper pelvis and knee thoroughly with Betadine or Chlorhexidine for a minimum of ten minutes. Drape with sterile cover. Administer appropriate prophylactic antibiotics to avoid nosocomial infections.

Pre Procedural Preparations: Pass contents of Bone Marrow Aspiration Pack to sterile field. Prepare a Heparinised Saline solution (2000 (VrnL) by transferring $10 \mathrm{ml}$ of Heparin $(5000 \mathrm{u} / \mathrm{mL})$ to $15 \mathrm{ml}$ of normal saline in a labeled beaker / basin placed in a sterile field. Rinse Aspiration Needles and Stylets, including needle lumen with heparinised saline. Return any remaining solution from the syringe to the beaker/basin.

Procedure: In posterior approach, place the patient in prone position palpate and locate the superior aspect of the posterior iliac crest. Prepare the surgical site and anaesthetize the area using sterile technique. Make a small stab incision for needle insertion. Prepare needle after removing the plastic guard from the aspiration needle, remove and reinsert the stylet one time to ensure that it can be easily separated from the cannula of the needle once it is inserted into the bone. To remove the stylet, rotate it $90^{\circ}$ counter clock wise and pull it straight out. Replace the stylet in the cannula and lock it back in place by rotating it $9 \mathrm{Q}^{\circ}$ clockwise so that it aligns with the lower portion of the needle handle. Grasp the bone marrow aspiration needle with the proximal end firmly seated in palm. Advance the needle using gentle but firm pressure. Rotate the needle in alternating clockwise counterclockwise motion or gently tap the needle with the mallet. Decreased resistance may be encountered upon penetration of the cortex and entrance into the marrow cavity. Remove the stylet by rotating the upper section of the handle counterclockwise and slowly pulling straight out. Screw syringe into hub Using sterile technique, screw the distal end of the included syringe into the proximal hub of the bone marrow aspiration needle. Ensure a firm fit, but do not over tighten. Withdraw the syringe plunger to draw 2-4 $\mathrm{ml}$ of bone marrow into the syringe chamber. If marrow still does not aspirate, reposition the needle slightly or remove reinsert the stylet and repeat steps 5 to 10 with a slight change in needle trajectory.

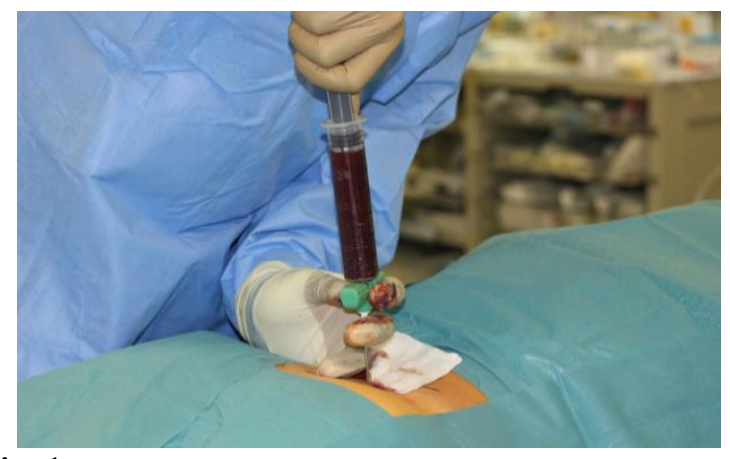

Fig. 1

To aspirate additional marrow penetrate deeper within the same site or use the fan technique to pull marrow from a new site using the same incision. If pulling additional marrow from deeper within the same site, advance the needle $1 \mathrm{~cm}$ along the same trajectory. The needle may also be rotated $90 \mathrm{G}$ to expose side holes to bone from which bone marrow has not yet been drawn. If using the fan technique, slightly withdraw the needle and reinsert it as described at a new trajectory along the same plane as the original insertion. Remove stylet. All syringes, cannula and stylets must be rinsed with heparinised saline solution between each use.

Marrow Transfer: After each aspiration, infuse all the contents of the $30 \mathrm{~mL}$ aspiration syringes immediately into the blood transfer bag and mix the contents so as to prevent the formation of any clots.

Transfer to Process Disposables (PD): Open clamp on the marrow filter unit port of the blood bag and attach a $60 \mathrm{~mL}$ syringe with fem/fem connector to the same. Using a sterile alcohol or any antiseptic swab, wipe the top of the reinjection port of the PD. Slowly dispense 60 $\mathrm{mL}$ bone marrow aspirate (BMA) into each of the marrow chamber of the PD through the redaccesssite.

Load Centrifuge: Place the Process Disposables(s) into the holders of the System. Insert the reusable balancer into the opposite bucket; balance weight (BW-60) for the $60 \mathrm{~mL}$ PD if centrifuging only one PD. Use appropriate Balance Weight when running one PD. Close lid on machine. The AMBER "LID OPEN" light must disappear. Press GREEN "START" button to start the process. Total processing time is approximately 14 minutes with 3200rpm.

Cell Resuspension: Use sterile alcohol or antiseptic swabs to wipe the top of the WHITE injection port of PD. Use the syringe with blunt Cannula and yellow spacers to withdraw unwanted plasma from the plasma 
chamber of PD. The remaining volume in the chamber is $10 \mathrm{~mL}$. Discard the plasma and the $30 \mathrm{~mL}$ syringe. Withdraw remaining plasma into and gently inject back and forth into the plasma chamber to resuspend the bone marrow cells into the plasma. Repeat above steps 2-3 times (until cells are visibly resuspended in the plasma) and withdraw total volume into syringe.

Observe base of plasma chamber to confirm all cells have been withdrawn into BMAC syringe. Transfer back into sterile field by connecting BMAC syringe to sterile luer lock connector on $20 \mathrm{~mL}$ sterile BMAC receiving syringe held by the scrub nurse in the sterile field. Dispense $0.5 \mathrm{ml}$ sample of the pooled concentrate from this syringe into the orange top tube for cell count analysis. Patient is placed in supine position knee is kept in flexion at 30-50 degree parts painted and draped 6$8 \mathrm{ml}$ of the prepared concentrate taken in a $10 \mathrm{ml}$ syringe and injection through lateral joint line. Post operatively NSAIDS given for 2 days, immediate pain relief. No medications or physiotherapy were given to any of patient during the study period.

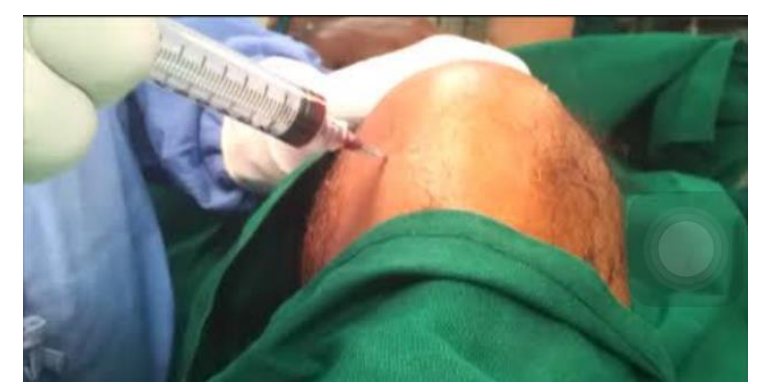

Fig. 2

Pre OP Evaluation: X-ray knee AP and lateral views, Visual analogue score, knee society score, WOMAC score, ESR, C-RP, RA Factor.

Post OP Evaluation after 6 Months: Visual analogue score, knee society score and WOMAC score.

\section{Results}

The total number of patients is 30 of which 19 $(63.3 \%)$ were female and $11(36 \%)$ were male. The age group of $50-55$ had $16(53.3 \%)$ patients and the age group of 56-60 had $14(46 \%)$ patients. In this $19(63.3 \%)$ patient presented with left knee osteoarthritis and 11 (36\%) patients right knee osteoarthritis. 19 patients presented with grade 2 osteoarthritis and 11 patients presented with grade 3 osteoarthritis.

Table 1 correlation of VAS Pain SCORE (Visual Analogue Score) preoperative and post-operative follow up at 6 months after BMAC injection- OA knee

\begin{tabular}{|l|c|c|c|}
\hline S. No & $\begin{array}{c}\text { VAS Pain } \\
\text { Score n= 30 }\end{array}$ & $\begin{array}{c}\text { Pre } \\
\text { Operative }\end{array}$ & $\begin{array}{c}\text { Post Operative } \\
\text { at 6 months }\end{array}$ \\
\hline 1 & $0-2$ & $4(13.3 \%)$ & $30(100 \%)$ \\
\hline 2 & $3-4$ & $26(86.6 \%)$ & 0 \\
\hline 3 & $>=5$ & 0 & 0 \\
\hline
\end{tabular}

Table 1 shows VAS pain score $86.6 \%$ were in score range of 3-4 preoperatively which decreased to score range of 0-2 100\% of the patients after BMAC Injection postoperatively at 6 months follow up. On Pre-operative evaluation of average of visual analog score was found to be 3.2 with minimum VAS score of 2 and maximum of 4. Postoperatively VAS score has reduced to average of 0.3 and with minimum of 0 and maximum of 1 . With $\mathrm{P}$ value $<0.001$ statistically significant.

Table 2: Correlation of KSS score (knee society score) preoperative and Post-operative follow up at 6 months after BMAC injection -OA knee

\begin{tabular}{|l|c|c|c|}
\hline S. No & $\begin{array}{c}\text { KSS score } \\
\text { range n =30 }\end{array}$ & Pre-operative & $\begin{array}{c}\text { Post-ore } \\
\text { operative at 6 } \\
\text { months }\end{array}$ \\
\hline 1 & $<60$ & $3(10 \%)$ & 0 \\
\hline 2 & $60-69$ & $17(56 \%)$ & 0 \\
\hline 3 & $70-79$ & $6(20 \%)$ & $4(13.3 \%)$ \\
\hline 4 & $80-100$ & $4(13.3 \%)$ & $26(86.6 \%)$ \\
\hline
\end{tabular}

Table 2 explains that the KSS Score preoperatively $56 \%$ in range of $60-69$ increased to $86.6 \%$ in range of 80 100 postoperatively at 6 months after BMAC injection. On Pre-operative evaluation of knee society score was found to be on an average of 68.5 with minimum of 56 and maximum of 80 . Which has post operatively improved to mean KSS score of 87 with minimum of 78 and maximum of 92 with $\mathrm{P}$ value of $<0.05$ statistically significant.

Table 3: correlation of Total WOMAC score Preoperative and Post- operative follow up at 6 months after BMAC injection

\begin{tabular}{|l|c|c|c|}
\hline S. No & $\begin{array}{c}\text { Total } \\
\text { WOMAC } \\
\text { score n= 30 }\end{array}$ & $\begin{array}{c}\text { Pre } \\
\text { operative }\end{array}$ & $\begin{array}{c}\text { Post } \\
\text { operative } \\
\text { at 6 months }\end{array}$ \\
\hline 1 & $<60$ & $6(20 \%)$ & 0 \\
\hline 2 & $61-70$ & $11(36.6 \%)$ & 0 \\
\hline 3 & $71-80$ & $13(50 \%)$ & 0 \\
\hline 4 & $81-90$ & 0 & $15(50 \%)$ \\
\hline 5 & $91-96$ & 0 & $15(50 \%)$ \\
\hline
\end{tabular}

Table 3 describes that $50 \%$ and $36.6 \%$ in total WOMAC score preoperative in the score range respectively 71-80 and 61-70.whereas the total WOMAC score range increased to 81-90 and 91-96 including $50 \%$ patients in each respectively. On Pre Operative evaluation of WOMAC score was found to be on an average of 69 with minimum of 52 and maximum of 80 . Which has post operatively improved to mean WOMAC score of 90.6 with minimum of 82 and maximum of 96 with significant $\mathrm{p}$ value $<0.001$. 


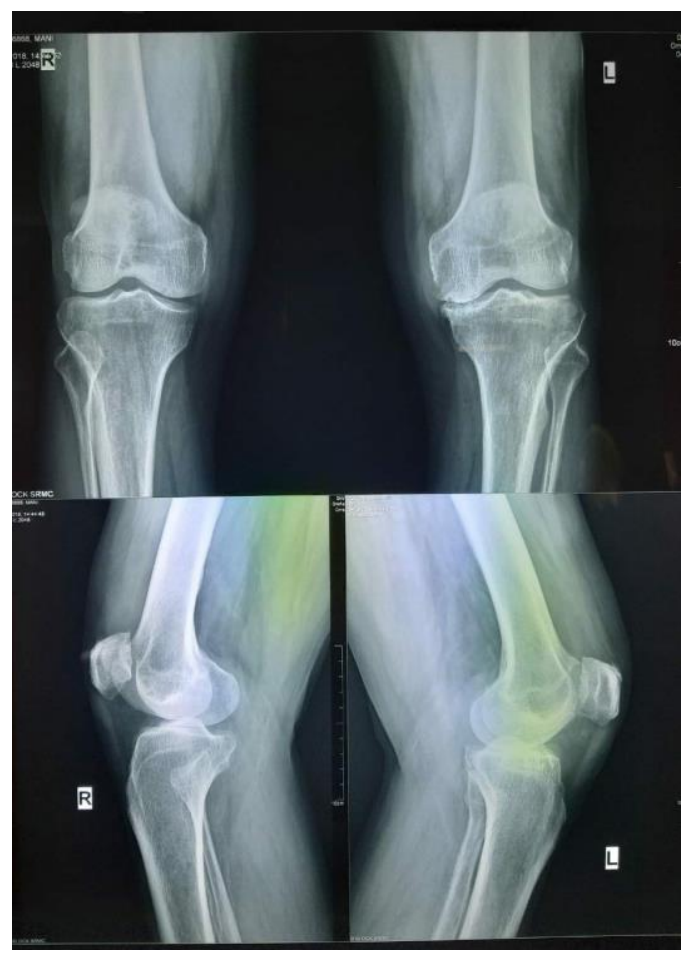

Fig. 3

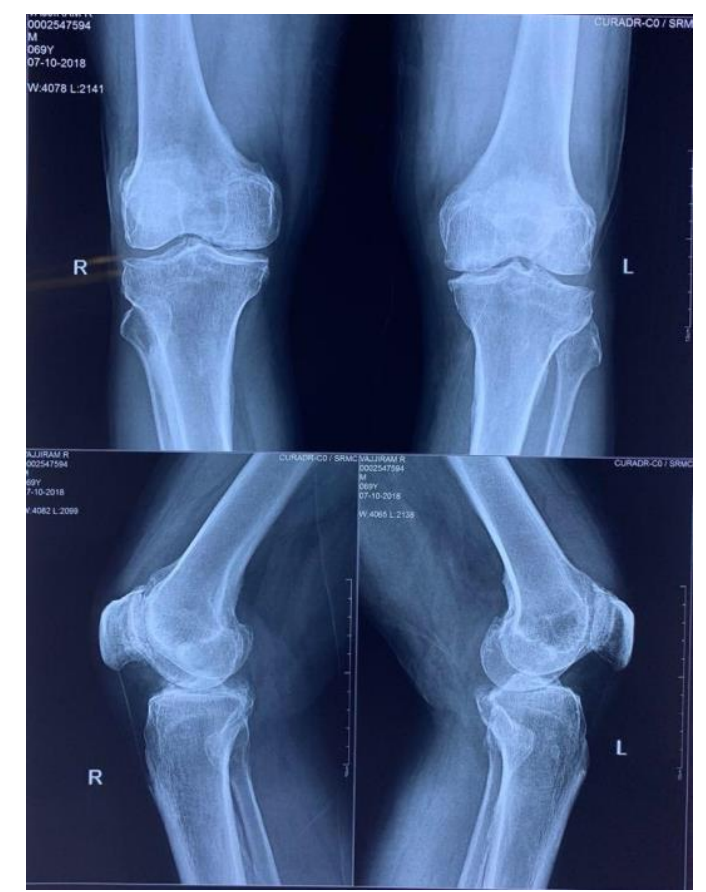

Fig. 4

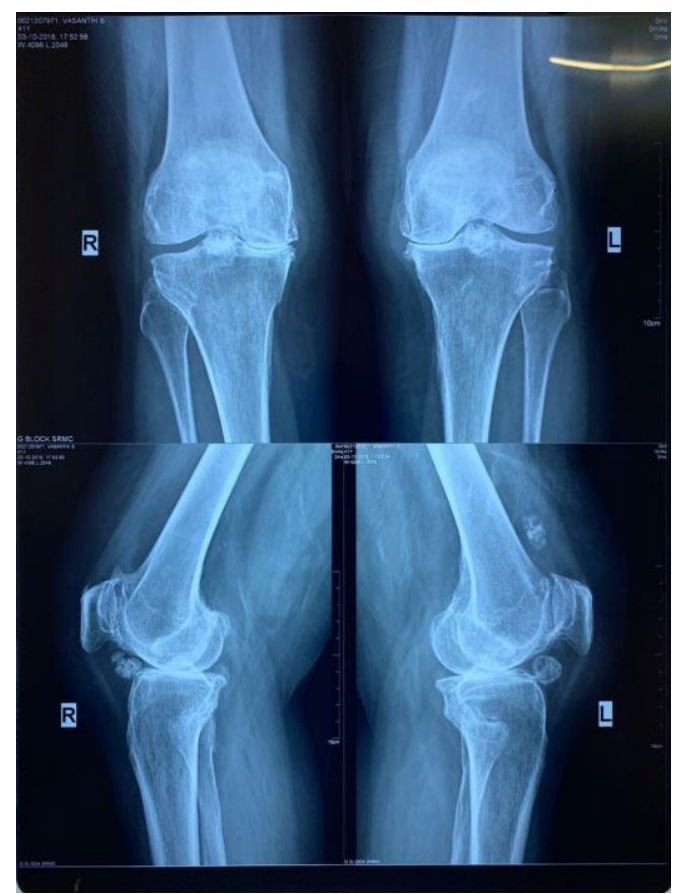

Fig. 5

\section{Discussion}

Osteoarthritis is characterized by catabolic and inflammatory joint environment. Thus the key to effective OA knee treatment is to promote cartilage regeneration and to reduce local inflammation. Owing to the above characteristics, mesenchymal stem cells (MSC's) have significant therapeutic potential. MSC's can be easily isolated from bone marrow, fat or synovial membrane. ${ }^{1}$

Current evidence based therapies focus on symptom improvement or total joint replacement versus prevention or improvement in the progressive destruction of OA joints. The concept of intra-articular delivery MSC's opens up novel treatment options for this disease process - OA knee. Many published recent studies support the feasibility, safety and efficacy of bone marrow derived MSC therapy in degenerative knee conditions including OA.

BMC has multiple beneficial contents for regeneration of tissue, including hematopoietic stem cells, platelet (growth factors) cytokines and chemokines. BMC also has anti-inflammatory, chondrogenic and immunogenic properties. All the above characteristics makes BMC as novel treatment for OA knee. Our study aims to study the efficacy and outcome of BMC injection OA knee.

In our study most of the patients who had OA knee were between 50-55 years of age, which was similar to Upadhyay $^{6}$ et al study 2014 . In this study $63.3 \%$ of female gender and $36.7 \%$ of male gender population which was similar to $\mathrm{Kim}^{7}$ et al study, 2014. Their study had $58.5 \%$ female and $41.5 \%$ male population.

This study had 30 patients enrolled, all them had unilateral OA knee. Of which $63.3 \%$ had left side OA 
Knee whereas $36.7 \%$ had right side OA knee. In 2013, Hauser and Orlofsky et $\mathrm{al}^{8}$ study of 7 case series of OA knee had 3 patients with left OA Knee, which was similar to our study. Our study assessed the degree of the OA knee by K-L grade (Kellgen- Lawrence grading scale) on standing Anteroposterior (AP) view. Kim et al study (4) done in 2014 had OA knee of grade I - 16\%, grade II $-32 \%$, grade III $-44 \%$ and $8 \%$ grade IV whereas in our study 8 patients $(60 \%)$ had grade II OA knee and 12 patients $(40 \%)$ had grade III OA knee.

Preoperative and postoperative follow up at 6 months following BMAC injection OA knee. Our study had preoperative VAS pain score had a range 3-4 for 26 patients $(86.7 \%)$ whereas 4 patients $(13.3 \%)$. There was marked decrease in VAS pain score following BMAC injection at postoperative follow up at 6 months to VAS range 0-2 had all 30 patients. In 2014, Kim et al study showed that the effect of BMAC was significant. The VAS score decreased from 8.2 preoperatively to 5.5,5.3 post operatively at 3,6 months showing more improving aspect in the group of grade I through III.

Regarding functional scoring Knee society score (KSS) and the western Ontario and Mc Master universities osteoarthritis Index ( WOMAC) are the two scoring system used in our study to assess the knee functional state preoperatively and postoperatively. Correlation of KSS score pre-operative and postoperative follow up at 6 months following BMAC injection - OA Knee. In our study preoperatively KSS score had maximum 17 patients $(56.7 \%)$ in the score range $60-69,6$ patients $(20 \%)$ in $70-79$ score range whereas 4 patients $(13.3 \%)$ in $80-100$ score range, 3 patients $(10 \%)$ in score range lesser than 60 . Following BMAC injection for OA knee at 6 months follow up $86.7 \%$ patients had excellent score range $80-100$ whereas $13.3 \%$ had score range $70-79$. Our study shows a statistical significant $\mathrm{p}$ value $<0.001$.

In 2014, Kim at el study used IKDC score (International Knee Documentation Commitee) and KOOS score for functional assessment of OA Knee after BMAC inection. Both IKDC and KOOS score showed marked increased in their score from pre-operatively 37.7 and 25.3 to 66.3 and 68.5 postoperatively at 6 months follow up which was similar to our study results. Correlation of Total WOMAC score pre-operative and post-operative follow up at 6 months following BMAC injection - OA Knee: Total WOMAC score assess the pain, stiffness and physical function of OA knee with total score of 96.

In our study - 13 patients (43.3\%) had score range $71-80,11$ patients $(36.7 \%)$ score range $61-70$ whereas 6 patients $(20 \%)$ had poor score lesser than 60 preoperatively. Post-operatively at 6 months follow up after BMAC injection OA knee $-50 \%$ had score range 91-96 and 50\% had score between 81- 90. The patients treated with BMAC showed statistically significant improvement $\mathrm{p}$ value $<0.05$ at 6 months follow up. In Upadhyay et al study done in 2014, total WOMAC score pre operatively mean $45.68+-7.163$ whereas at 6 months follow up 18.45+-9.361. Our study results were similar to Upadhyay et al study.

\section{Study Limitations}

In our study we were not able to assess the strength of this cartilage in the knee joint due to lack of pre-op procedure MRI Imaging and Post-op Procedure MRI Imaging. In our study we assessed the efficacy of the bone marrow aspirate concentrate in the early stage of OA knee (type 2 and 3 ) only and we did not compare the efficacy of other therapy (Inj. depomedrol, PRP Injection and Physiotherapy).

\section{Conclusion}

The use of Bone marrow concentrate injection (BMAC) in Osteoarthritis of knee was found to be more efficacious with good clinical and functional outcome. Hence Bone marrow aspirate concentrate may be considered as one of the alternative therapeutic option for Early Osteoarthritis of knee.

\section{References}

1. Chen D, Shen J, Zhao W, Wang T, Han L, Hamilton JL, et al. Osteoarthritis: toward a comprehensive understanding of pathological mechanism. Bone Res. 2017;5:16044

2. Lespasio M, Piuzzi NS, Husni ME. Knee osteoarthritis: a primer. Perm J. 2017;21:16-183.

3. Heidari B. Knee osteoarthritis prevalence, risk factors, pathogenesis and features: part I. Caspian J Intern Med. 2011;2:205-212.

4. N. Arden, M.C. Nevitt Osteoarthritis: epidemiology. Best Pract Res Clin Rheumatol. 20 (1) (2006 Feb), pp. 3-25

5. Betsch M, Thelen S, Santak L. The role of erythropoietin and bone marrow concentrate in the treatment of osteochondral defects in mini-pigs. PLoS One. 2014;9:e92766.

6. Upadhyay S, Varma HS, Chaurasia S, Chansoria M. Randomized blinded comparative analysis of autologous mononuclear medullary fraction and viscosupplementation in grade 0 and grade 1 radiographic scale of knee osteoarthritis. Indian J Pain. 2014;28:13442.

7. Kim, JD., Lee, G.W., Jung, G.H. Eur J Orthop Surg Traumatol. 2014;24:1505. https://doi.org/10.1007/s00590-013-1393-9.

8. Hauser RA, Orlofsky A. Regenerative Injection Therapy with Whole Bone Marrow Aspirate for Degenerative Joint Disease: A Case Series. Clinical Medicine Insights Arthritis and Musculoskeletal Disorders. 2013;6:65-72. doi:10.4137/CMAMD.S10951.

How to cite this article: Ajees J. T, Mohan K. M.
Efficacy and functional outcome of bone marrow
aspirate concentrate injection in osteoarthritis of knee-
A prospective study. Indian J Orthop Surg.
2018;4(4):320-324.

